\title{
Human hair shaft proteomic profiling: Individual differences, site specificity and cuticle analysis
}

Hair from different individuals can be distinguished by physical properties. Although some data exist on other species, examination of the individual molecular differences within the human hair shaft has not been thoroughly investigated. Shotgun proteomic analysis revealed considerable variation in profile among samples from Caucasian, African-American, Kenyan and Korean subjects. Within these ethnic groups, prominent keratin proteins served to distinguish individual profiles. Differences between ethnic groups, less marked, relied to a large extent on levels of keratin associated proteins. In samples from Caucasian subjects, hair shafts from axillary, beard, pubic and scalp regions exhibited distinguishable profiles, with the last being most different from the others. Finally, the profile of isolated hair cuticle cells was distinguished from that of total hair shaft by levels of more than 20 proteins, the majority of which were prominent keratins. The cuticle also exhibited relatively high levels of epidermal transglutaminase (TGM3), accounting for its observed low degree of protein extraction by denaturants. In addition to providing insight into hair structure, present findings may lead to improvements in differentiating hair from various ethnic origins and offer an approach to extending use of hair in crime scene evidence for distinguishing among individuals. 
2 Chelsea N. Laatsch $\dagger$, Blythe P. Durbin-Johnson\#, David M. Rocke\#, Sophie Mukwana§, Abby

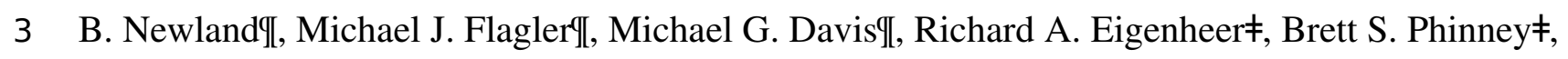

4 Robert H. Rice†*

$5 \dagger$ Forensic Science Graduate Program and Department of Environmental Toxicology, University

6 of California, Davis

7 \#Division of Biostatistics, Department of Public Health Sciences, Clinical and Translational

8 Science Center Biostatistics Core, University of California, Davis

9 §Biotech Forensics, Nairobi, Kenya

10 IProcter \& Gamble, Mason Business Center, Mason, $\mathrm{OH}$

11 ‡Proteomics Core Facility, University of California, Davis

$12 *$ Corresponding Author. Department of Environmental Toxicology, One Shields Avenue,

13 University of California, Davis, CA 95616-8588; Tel 530-752-5176; Fax 530-752-3394, Email

14 rhrice@ucdavis.edu

15 Keywords: Ancestry, Body site, Corneocytes, Differential expression, Forensic evidence,

16 Keratins, Keratin associated proteins, Transglutaminase 


\section{INTRODUCTION}

18 Mass spectrometry coupled with database searching now permits identification of many proteins

19 in complex structures such as hair shaft (Lee et al, 2006; Barthélemy et al, 2012). In addition to

20 its high keratin content, hair is challenging to analyze in part due to the extensive

21 transglutaminase-mediated isopeptide cross-linking that prevents solubilization of $\approx 15 \%$ of the

22 constituent protein even by strong denaturants under reducing conditions. Since only $\approx 20 \%$ of the

23 lysines participate in isopeptide bonding, generation of peptides by trypsin fragmentation permits

24 efficient identification of the cross-linked as well as non-cross-linked protein and comparison of

25 relative amounts in parallel samples. The cross-linked proteins, a sampling of those present in

26 corneocytes, include those capable of being solubilized and are derived from the various cellular

27 compartments.

28 The structure of the hair shaft is complex, with corneocytes of the cuticle, cortex and

29 medulla exhibiting distinct features readily visible ultramicroscopically after extraction with

30 strong protein denaturants such as sodium dodecyl sulfate (SDS) under reducing conditions (Rice

31 et al, 1994). For example, cuticle cell protein appears much less extractable than that of the

32 cortex, attributed to a higher level of isopeptide bonding (Zahn et al, 1980). Subfractionation of

33 the cuticle by physical and enzymatic methods demonstrated different amino acid compositions

34 indicative of different protein compositions (Swift and Bews, 1974a). A comprehensive analysis

35 of cuticle from wool fibers clarified that it contains abundant keratins and keratin-associated

36 proteins (KAPs) as well as a variety of structural proteins and enzymes, similar to human hair

37 (Koehn et al, 2010).

38 Previous analyses reported in the literature using hair clipped from inbred mouse strains

39 suggest that humans might show differences among individuals or according to ancestry (Rice et 
40 al, 2009; Rice et al, 2012). This possibility is consistent with observations that the large majority

41 of genes are differentially expressed among individual humans and that a smaller fraction varies

42 among specific populations (Lappalainen and Dermitzakis, 2010). Present work explores whether

43 a limited survey of hair samples from several ethnic groups is sufficient to reveal such variation,

44 and whether hair shafts from the scalp are distinguishable from other anatomic locations. It also

45 explores whether the cuticle has a distinctive protein profile compared to intact hair shaft.

\section{MATERIALS AND METHODS}

\section{Hair samples}

48 Samples of scalp and body hair were collected from unrelated volunteer adult subjects (ages 20-

49 65) with written consent approved by the University of California Davis Institutional Review

50 Board (protocol 217868). Samples were collected from individuals whose hair was not

51 chemically treated (dyed, bleached, straightened). Scalp hair from Caucasian (six male), African-

52 American (five male), Korean (three male, two female) and Kenyan (four male, one female)

53 individuals were analyzed. Three samples from each subject were analyzed; in most cases, scalp

54 hair was collected at the same time and processed from three sites on the head (left, right, center).

55 For analysis of cuticle, several grams of hair were collected from participating individuals (five

56 Caucasian males, two Asian females) during a haircut for single analyses. The parents of Korean

57 and Kenyan subjects were also Korean or Kenyan, respectively. Samples of axillary, facial

58 (beard) and pubic hair were collected from male subjects.

59 Cuticle Isolation

60 Hair samples were wetted with distilled water, rinsed with clarifying shampoo and then rinsed

61 with water several times with gentle swirling. Initial samples $(0.2 \mathrm{~g})$ were transferred to glass

62 scintillation vials, suspended in $2 \mathrm{ml}$ of distilled water and shaken at 2,500 rpm in a DVX-2500 
63 Multi-tube Vortexer (VWR, Radnor, PA). After various lengths of time, suspended cells were

64 recovered from the water fraction by centrifugation. One aliquot was submitted for protein

65 determination and another was examined by scanning electron microscopy after air drying and

66 coating with $\mathrm{Au} / \mathrm{Pd}$ in a Hitachi S-4700 instrument at $3 \mathrm{Kv}$ with built in PCI image software.

67 Purity of the cuticle cells was estimated by visual inspection. Larger aliquots of hair were then

68 processed together at the empirically determined optimal time period.

\section{Sample processing}

70 Samples (2-4 mg) were rinsed twice in 2\% SDS - 0.1 M sodium phosphate ( $\mathrm{pH} 7.8$ ) and

71 incubated in $0.4 \mathrm{ml}$ of this buffer containing $25 \mathrm{mM}$ dithioerythritol for disulfide reduction and

72 then alkylation with iodoacetamide. Proteins were recovered as a flocculent precipitate by

73 centrifugation after addition of $1 \mathrm{ml}$ of ethanol, rinsed twice with $67 \%$ ethanol, once with freshly

74 prepared $0.1 \mathrm{M}$ ammonium bicarbonate and digested for 3 days with daily additions of $40 \mu \mathrm{g}$ of

75 stabilized trypsin (Rice et al, 1977). Clarified digests, containing $90 \%$ of the digested protein,

76 were submitted for mass spectrometric analysis. Alkylation of the protein with 2-

77 bromoethylamine instead of iodoacetamide provided marginally higher peptide yields for many

78 proteins but largely suppressed identification of keratin-associated proteins as previously reported

79 (Rice et al, 2012).

80 Mass spectrometry and protein identification

81 Samples adjusted to approximately equal peptide amounts by $\mathrm{A}^{280}$ and adjusted to $0.5 \%$

82 trifluoracetic acid were directly loaded onto an Agilent ZORBAX 300SB C18 reverse-phase trap

83 cartridge which, after loading, was switched in-line with a Michrom Magic C18 AQ $200 \mu \mathrm{m} x$

$84150 \mathrm{~mm}$ nano-LC column connected to a Thermo-Finnigan LTQ iontrap mass spectrometer

85 through a Michrom Advance Plug and Play nanospray source with CTC Pal autosampler. The

86 nano-LC column was used with a binary solvent gradient; buffer A was composed of $0.1 \%$ 
87 formic acid and buffer B composed of $100 \%$ acetonitrile. The 120 min gradient consisted of the

88 steps 2-35\% buffer B for $85 \mathrm{~min}, 35-80 \%$ buffer B for $23 \mathrm{~min}$, hold for $1 \mathrm{~min}, 80-2 \%$ buffer B

89 for $1 \mathrm{~min}$, then hold for $10 \mathrm{~min}$, at a flow rate of $2 \mu \mathrm{l} / \mathrm{min}$ for maximal separation of tryptic

90 peptides. An MS survey scan was obtained for the m/z range 375-1400, and MS/MS spectra were

91 acquired from the 10 most intense ions in the MS scan by subjecting them to automated low

92 energy CID. An isolation mass window of 2 Da was used for the precursor ion selection, and

93 normalized collision energy of $35 \%$ was used for the fragmentation. A 2 min duration was used

94 for the dynamic exclusion. Monitoring of column washes indicated negligible intersample

95 contamination.

96 Tandem mass spectra were extracted with Xcalibur version 2.0.7. All MS/MS samples

97 were analyzed using X!Tandem (The GPM, thegpm.org; version CYCLONE (2012.10.01.2)). X!

98 Tandem was set up to search a 2012 Uniprot human database appended to a database of common

99 non-human contaminants (cRAP, http://www.thegpm.org/crap/), both of which were appended to

100 an identical but reversed database for calculating false discovery rates (136,252 proteins total),

101 assuming the digestion enzyme was trypsin. X!Tandem was searched with a fragment ion mass

102 tolerance of $0.40 \mathrm{Da}$ and a parent ion tolerance of $1.8 \mathrm{Da}$. Iodoacetamide derivative of cysteine

103 was specified in X!Tandem as a fixed modification. Deamidation of asparagine and glutamine,

104 oxidation of methionine and tryptophan, sulfone of methionine and tryptophan oxidation to

105 formylkynurenin of tryptophan were specified in X!Tandem as variable modifications. Scaffold

106 version_4.2.1 (Proteome Software Inc., Portland, OR) was used to validate MS/MS based peptide

107 and protein identifications. Peptide identifications were accepted if they could be established at

108 greater than $90 \%$ probability as specified by the Peptide Prophet algorithm (peptide decoy false

109 discovery rate $0.2 \%$ ) (Keller et al, 2002). Protein identifications were accepted if they could be 
110

111 decoy false discovery rate 4.1\%). Protein probabilities were assigned by the Protein Prophet

112 algorithm (Nesvizhskii et al, 2003). Numbers of distributed spectral counts (called weighed

113 spectral counts in Scaffold) were tabulated using experiment-wide grouping. The Scaffold file

114 containing all the peptide data used in the analysis is now available in the public database on the

115 MassIVE repository (ID: MSV000078650; http://massive.ucsd.edu).

116 Because certain keratins are well known to contain identical peptides, we used a

117 distributed spectral count (called weighed spectral count in Scaffold) to model spectral counts

118 more accurately across the proteins identified in this study. Scaffold's weighed spectral counts

119 appropriates a percentage of each count divided among the protein groups that share that peptide.

120 The formulation for that percentage for peptide(i) assigned to protein(j) is

$121 \mathrm{PPS}(\mathrm{j}) / \mathrm{sum}(\operatorname{PPS}(1 \ldots \mathrm{n}))$, where $\operatorname{PPS}(\mathrm{j})$ is the sum of the peptides $(1 \ldots \mathrm{m})$ for protein $(\mathrm{j})$ and $(1 \ldots \mathrm{n})$ is

122 the set of proteins that contain peptide(i). The weighted spectrum count is the sum of those

123 percentages for each protein group. This is similar to method 3-a in Zhang et al (2010), but

124 Scaffold uses the summed probabilities as normalizers instead of summed exclusive counts

125 (Brian Searle, Proteome Software, pers. comm., 2014). In addition, every protein identified was

126 required to have at least one unique peptide that was not shared with any other protein. 


\section{Statistical analysis}

128 Total hair and cuticle samples from different subjects were compared using mixed-effects

129 overdispersed Poisson regression models, including a fixed effect for total hair vs. cuticle, a fixed

130 effect for ethnicity (to adjust for imbalances between total hair and cuticle donors) and a random

131 effect for subject. This analysis included scalp total hair samples from one Caucasian subject,

132 five Kenyan subjects, five African American subjects, and five Korean subjects; cuticle samples

133 were from five white subjects and two Asian subjects.

134 Hair from different sites (scalp, axillary, facial, and pubic) were likewise compared within

135 subjects using mixed-effects overdispersed Poisson regression models, including a fixed effect

136 for site and a random effect for subject. Pairwise comparisons between sites were conducted

137 using the Tukey HSD method. This analysis included scalp, axillary, facial, and pubic hair

138 samples from four Caucasian subjects.

139 Scalp samples were compared among subjects of the same ethnicity using an

140 overdispersed Poisson model, treating subject as a fixed effect.

141 To include proteins expressed at very low levels, a different analysis approach was taken

142 for the comparison of hair and cuticle samples within subjects. Normalization factors were

143 calculated using the Trimmed Mean of M-Values (TMM) method developed for RNA-Seq data

144 (Robinson and Oshlack, 2010). Counts were then divided by these normalization factors. These

145 preprocessing steps were conducted once for the entire data set and not repeated for individual

146 analyses.

147 Total hair and cuticle samples were then compared within subjects using mixed-effects

148 lognormal-Poisson regression models (similar to negative binomial models), including a fixed

149 effect for total hair vs. cuticle and a random effect for subject. This analysis included scalp total 
150 hair and cuticle samples from five Caucasian subjects. Mixed effects lognormal-Poisson

151 regression modeling was conducted using the lme4 package, version 0.999999-2 (Bates et al,

152 2013) in the statistical software environment R, version 3.0.1 (R Core Team, 2013).

153 Error bars in figures represent 95\% confidence intervals for parameter estimates from the models

154 described above. Hierarchical clustering was performed with the hclust function in the R

155 statistical software environment using the complete linkage method as described in

156 http://nlp.stanford.edu/IR-book/html/htmledition/single-link-and-complete-link-clustering-1.html

157 (Manning et al, 2008).

158 RESULTS

159 Distinguishing among hair shaft samples within ethnic groups

160 Scalp hair samples from Caucasian, African-American, Korean and Kenyan individuals were

161 analyzed. Analysis of triplicate samples from each subject (one each from different regions of the

162 scalp) provided assurance that the differences among samples reflected individual subject

163 differences. Considerable variation was evident in the profiles of prevalent proteins within each

164 ethnic group. Using pairwise comparisons of 76 proteins present in sufficient amounts, each

165 group was analyzed separately (Supplemental Tables S2-S5). As shown in Table 1, samples from

166 individuals were distinguishable in each group, although the number of significant protein

167 differences varied greatly. Figure 1 displays for the Caucasian group nine proteins that were

168 present at significantly different levels in comparisons among at least some of the 6 individuals

169 analyzed. In such analyses, K32, K33B, K34 and K83 exhibited significant differences in at least

$17040 \%$ of the pairwise comparisons within all four ethnic groups, while K31, K35, K39 and K86

171 exhibited similarly high inter-subject variability in at least three of the ethnic groups. 


\section{Distinguishing samples from different ethnic groups}

173 In contrast to the high variation in protein expression within ethnic groups, consistent differences

174 among ethnic groups were less marked. As shown in Table 2, the groups were distinguishable

175 (Supplemental Table S6), with African-American samples being the most distinctive. Most useful

176 for pairwise comparisons were KAPs, accounting for 21 of the 32 significant differences (66\%)

177 observed overall. Illustrated in Fig. 2, KAPs 2-4, 4-3, 13-1 and 13-2 accounted for 16 (50\%),

178 whereas K40, selenium binding protein-1 (SBP1) and epidermal transglutaminase (TGM3)

179 accounted for seven $(22 \%)$ of the total significant differences.

180 Distinguishing hair shafts from different body sites

181 Hair samples from three body sites (axillary, facial and public regions) were analyzed and

182 compared to those from scalp. As seen in Fig. 3, pairwise comparisons using 92 proteins

183 permitted distinguishing among them (Supplemental Table S7). Scalp hair displayed the most

184 differences from the others, while axillary and pubic hair displayed the fewest differences from

185 each other. In these comparisons, KRTs and KAPs together and in equal amounts accounted for

186 only one-third of the significant differences. A variety of enzymes and structural proteins

187 contributed to the observed differences as illustrated in Fig. 4.

\section{Cuticle analysis}

189 The yield of cuticle cells from hair fibers increased with time of agitation in water as previously

190 described (Swift and Bews, 1974b). After several hours, however, the purity of the released

191 cuticle material was observed to decline as fragments from other parts of the hair shaft increased

192 in amount. Since the optimal time varied among subjects, treatment of each sample was

193 monitored by scanning electron microscopy to ensure its quality. The yield of purified cuticle

194 cells was estimated as $0.1 \%$ of the total hair mass. Samples submitted for proteomic analysis 
195 were estimated to be $>95 \%$ cuticle cells, with only rare non-cuticle cells being distinguishable, as 196 illustrated in Fig. 5.

197 The protein profiles of cuticle samples were distinctly different from those of the total 198 hair. When samples of both were compared from five Caucasian subjects, 34 proteins were seen 199 to be differentially expressed (Supplemental Table S8A). An additional comparison was made 200 between the cuticle samples from five Caucasian and two Asian subjects with total hair from 201 Caucasian, Korean, Kenyan and African-American subjects. In that case, 30 proteins were seen to 202 be differentially expressed (Supplemental Table S8B). Of these, 24 overlapped in the two 203 comparisons and were taken to be the most reliable indicators of differential expression. Of these, 204 more than half were KRTs, with six higher (K1, K2, K10, K32, K40, K82) and eight lower (K31, 205 K33A, K33B, K38, K39, K83, K85, K86) in the cuticle. Cuticle also displayed an elevated level 206 of TGM3, consistent with the relatively low degree of protein extraction by detergent compared 207 to the cortex (Rice et al, 1994). Other proteins enriched in cuticle were AIM1, TUBB2A and 208 VSIG8, while DSG4, DSP, KAP3-1, HIST1H2AG, HIST1H2BK and SFN were higher in total 209 hair shaft. Figure 6 illustrates 16 of the most distinctive proteins.

210 DISCUSSION

211 Human corneocyte protein profiling, as conducted in this work, has provided insight into 212 phenotyping the skin disease ichthyosis (Rice et al, 2013) and understanding the toxic response of

213 cultured keratinocytes to the environmental toxicant 2,3,7,8-tetrachlorodibenzo-p-dioxin (Hu et

214 al, 2013). Extending such efforts to compare hair shaft protein profiles among individuals has

215 several possible applications. As previously demonstrated, the profiles can reflect structural

216 features that may be pertinent to hair phenotype (Rice et al, 2009; Rice et al, 2012). This

217 approach could now be extended specifically to the cuticle in cases of anomalous structure of that 
218 layer (Rice et al, 1999a). Monitoring of hair protein profiles could be informative with respect to

219 keratin-related disease (Schweizer et al, 2007; Moll et al, 2008) and, since the hair shaft exhibits

220 a number of proteins with important functions elsewhere in the body, it could have more general

221 diagnostic utility. In such work, knowledge of the range of individual variability will be valuable

222 in analyzing profiling datasets. The relative paucity of proteins distinguishing hair from different

223 ethnic origins suggests that some visible differences (e.g., curliness) are not bestowed by major

224 structural proteins, and the importance of lipid processing for features such as combability is now

225 appreciated (Shimomura et al, 2009).

An ability to distinguish individuals by means of hair could be applicable to crime scenes,

227 where this is a commonly recovered type of evidence. Microscopic examination of hair evidence

228 can take into account features such as pigmentation (granule distribution and density, spectral

229 analysis), cosmetic treatment (dye, bleach), diameter, appearance in cross-section and structural

230 abnormalities of the shaft. Using all these features permits useful discrimination whether a given

231 hair originates from a specific individual (Gaudette, 1999), leading to the recommendation that

232 hair analysis should begin with a thorough microscopic examination (Scientific Working Group

233 on Materials Analysis, 2009). Nevertheless, the search for more objective criteria by which to

234 judge hair matches continues (Taupin, 2004). Hair evidence is ordinarily supplemented by DNA

235 evidence whenever possible (Rowe, 2001). When follicle tissue is present, nuclear DNA

236 extracted from a hair sample may identify the donor. In the great majority of cases, only

237 mitochondrial DNA (mtDNA) from the shaft is available. mtDNA can provide valuable

238 exclusionary evidence, but is not sufficient alone for individual identification. The information

239 from proteomic characterization is complementary to that from microscopic examination and

240 DNA analysis and may assist in discerning its body site of origin, thereby augmenting its 
241 evidentiary value. In present work, samples were subjected to pairwise comparison using

242 expression levels of specific protein components. Further effort may permit development of a

243 statistical classification scheme important in establishing a searchable database.

245 was clearly manifest in present results. The major hair shaft components, KRTs and KAPs, were

246 both useful in discriminating among individuals, but the latter appeared to be more useful among

247 ethnic groups. A previous study also pointed to differences in KAPs among subjects of African,

248 Jamaican and African-American origin (Porter et al, 2009). These findings, and the frequent

249 length polymorphisms they display (Fujikawa et al, 2012), often reflecting amino acid repeats in

250 the coding region, may impel further focus on KAPs for this purpose.

A strong genetic component appears to be involved in expression differences observed in

252 other species. This likely reflects variation in transcription factor binding affinity for chromatin

253 that can even have epigenetic consequences (Kasowski et al, 2013; Kilpinen et al, 2013;

254 McVicker et al, 2013). However, indications that the profile in humans could depend on age

255 (Giesen et al, 2011) and the possible influence of other factors including environment and diet

256 (Almeida et al, 2014) merit further study. In addition, how well cuticle cells remain attached to

257 the shaft could influence the profile. The latter could depend on hair length, weathering, and

258 cuticle cell stability, variables among individuals (Rice et al, 1999a). These factors would need

259 clarification in determining the value a database of individual hair profiles would have for

260 forensic science. Nevertheless, results so far provide a basis for further investigation.

261 TGM3 is known to be expressed in the cuticle and cortex of human hair shaft (Thibaut et

262 al, 2005). Emphasizing that cuticle exhibits intriguing differences in protein profile from cortex

263 (Koehn et al, 2010), present results provide a rationale for its proposed high content of isopeptide 
264 bonding in its relatively high expression level of TGM3. This could contribute to the persistence

265 of clearly demarcated endocuticle, exocuticle and marginal band (A) layers visible even after

266 detergent extraction (Rice et al, 1994), and is consistent with the severe structural defects (e.g.,

267 easier cuticle loss) due to TGM3 ablation (John et al, 2012). Not detected at such high levels in

268 present work, TGM1 is known to be important for stabilization of human cuticle cells, since

269 detergent extraction greatly perturbs their structure in individuals lacking this activity (Rice et al, 270 1999b).

271 Present results are complementary to exquisite immunochemical and in situ hybridization

272 studies of keratin expression in various layers of the hair follicle (Moll et al, 2008; Langbein et al,

273 2010). They permit identification of a variety of such proteins and others that are not KRTs and

274 KAPs, without the need for isoform-specific antibodies, and are applicable to the mature hair

275 shaft instead of the living cells. Similar to results with wool cuticle (Koehn et al, 2010), the

276 analysis revealed several epidermal keratins including K1 and K10 previously identified in

277 human hair cuticle (Stark et al, 1990). This unexpected finding appears to be in contrast to the

278 above reviews concerning hair follicle, but is difficult to attribute to contamination of the purified

279 preparations or to mis-identification due to peptides shared with other keratins. Further

280 investigation may help resolve this dichotomy.

\section{Acknowledgments}

282 We thank Dr. Ying L. Boissy for performing scanning electron microscopy.

\section{Supporting Information}

284 Supplemental Table S1. Profiles of protein constituents in samples of hair and isolated cuticle 
285 Supplemental Tables S2. Pairwise comparisons among Caucasian samples

286 Supplemental Table S3. Pairwise comparisons of African American samples

287 Supplemental Table S4. Pairwise comparisons of Kenyan samples

288 Supplemental Table S5. Pairwise comparisons of Korean samples

289 Supplemental Table S6. Pairwise comparisons of samples of different ethnic origin

290 Supplemental Table S7. Pairwise comparisons of samples from different body sites among 291 Caucasian subjects

292 Supplemental Table S8. Statistical testing of cuticle versus total hair shaft

\section{REFERENCES}

294 Almeida AM, Plowman JE, Harland DP, Thomas A, Kilminster T, Scanlon T, Milton J, 295 Greeff J, Oldham C, Clerens S (2014) Influence of feed restriction on the wool proteome: A 296 combined iTRAQ and fiber structural study. Journal of Proteomics 103:170-177

297 Barthélemy NR, Bednarczyk A, Schaeffer-Reiss C, Jullien D, Van Dorsselaer A, Cavusoglu 298 N (2012) Proteomic tools for the investigation of human hair structural proteins and evidence of 299 weakness sites on hair keratin coil segments. Analytical Biochemistry 421:43-55

$300 \quad$ Bates D, Maechler M, Bolker B (2013) lme4: Linear mixed-effects models using S4 classes. 301 R package version 0.999999-2. http://CRAN.R-project.org/package=lme4

302 Fujikawa H, Fujimoto A, Farooq M, Ito M, Shimomura Y (2012) Characterization of the 303 human hair keratin-associated protein 2 (KRTAP2) gene family. Journal of Investigative 304 Dermatology 132:1806-1813

305 Gaudette BD (1999) Evidential value of hair examination. Forensic Examination of Hair 306 (Robertson J, ed) Taylor \& Francis, London, pp 243-260 
308 processes influence keratin and KAP expression in human hair follicles. Experimental

309 Dermatology 20:759-761

310 Hu Q, Rice RH, Qin Q, Phinney BS, Eigenheer RA, Bao W, Zhao B (2013) Proteomic

311 analysis of human keratinocyte response to 2,3,7,8-tetrachlorodibenzo-p-dioxin (TCDD)

312 exposure. Journal of Proteome Research 12:5340-5347

313 John S, Thiebach L, Frie C, Mokkapati S, Bechtel M, Nischt R, Rosser-Davies S, Paulsson

314 M, Smyth N (2012) Epidermal transglutaminase (TGase 3) is required for proper hair

315 development, but not the formation of the epidermal barrier. PLoS One 7(4):e34252

316 Kasowski M, Kyriazopoulou-Panagiotopoulou S, Grubert F, Zaugg JB, Kundaje A, Liu Y,

317 Boyle AP, Zhang QC, Zakharia F, Spacek DV, Li J, Xie D, Olarerin-George A, Steinmetz LM,

318 Hogenesch JB, Kellis M, Batzoglou S, Snyder M (2013) Extensive variation in chromatin states

319 across humans. Science 342:750-752

320 Keller A, Nesvizhskii AI, Kolker E, Aebersold R (2002) Empirical statistical model to

321 estimate the accuracy of peptide identifications made by MS/MS and database search. Analytical

322 Chemistry 74:5383-5392

323 Kilpinen H, Waszak SM, Gschwind AR, Raghav SK, Witwicki RM, Orioli A, Migliavacca

324 E, Wiederkehr M, Gutierrez-Arcelus M, Panousis NI, Yurovsky A, Lappalainen T, Romano-

325 Palumbo L, Planchon A, Bielser D, Bryois J, Padioleau I, Udin G, Thurnheer S, Hacker D, Core

326 LJ, Lis JT, Hernandez N, Reymond A, Deplancke B, Dermitzakis ET (2013) Coordinated effects

327 of sequence variation on DNA binding, chromatin structure, and transcription. Science 342:744$328 \quad 747$ 
330 proteome of the wool cuticle. Journal of Proteome Research 9:2920-2928

331 Langbein L, Yoshida H, Praetzel-Wunder S, Parry DA, Schweizer J (2010) The keratins of 332 the human beard hair medulla: the riddle in the middle. Journal of Investigative Dermatology $333130: 55-73$

334 Lappalainen T, Dermitzakis ET (2010) Evolutionary history of regulatory variation in 335 human populations. Human Molecular Genetics 19:R197-R203

336 Lee YJ, Rice RH, Lee YM (2006) Proteome analysis of human hair shaft: From protein 337 identification to posttranslational modification. Molecular and Cellular Proteomics 5:789-800 338 Manning CD, Raghavan P, Schütze H (2008) Introduction to Information Retrieval, 339 Cambridge University Press

340 McVicker G, van de Geijn B, Degner JF, Cain CE, Banovich NE, Raj A, Lewellen N, 341 Myrthil M, Gilad Y, Pritchard JK (2013) Identification of genetic variants that affect histone 342 modifications in human cells. Science 342:747-749

343 Moll R, Divo M, Langbein L (2008) The human keratins: biology and pathology.

344 Histochemistry and Cell Biology 129:705-733

345 Nesvizhskii AI, Keller A, Kolker E, Aebersold R (2003) A statistical model for identifying 346 proteins by tandem mass spectrometry. Analytical Chemistry 75:4646-4658

347 Porter CE, Dixon F, Khine CC, Pistorio B, Bryant H, de la Mettrie R (2009) The behavior of 348 hair from different countries. Journal of Cosmetic Science 60:97-109

349 R Core Team (2013) R: A language and environment for statistical computing. 350 http://www.R-project.org/ 
352 methylation. Biochimica et Biophysica Acta 492:316-321

353 Rice RH, Wong VJ, Pinkerton KE (1994) Ultrastructural visualization of cross-linked

354 protein features in epidermal appendages. Journal of Cell Science 107:1985-1992

355 Rice RH, Wong VJ, Pinkerton KE, Sundberg JP (1999a) Cross-linked features of mouse

356 pelage hair resistant to detergent extraction. The Anatomical Record 254:231-237

357 Rice RH, Wong VJ, Williams ML, Price VH, Hohl D, Sundberg JP, Pinkerton KE (1999b)

358 Hair shaft defects visualized after detergent extraction. Experimental Dermatology 8:308-310

359 Rice RH, Rocke DM, Tsai H-S, Lee YJ, Silva KA, Sundberg JP (2009) Distinguishing

360 mouse strains by proteomic analysis of pelage hair. Journal of Investigative Dermatology

$361 \quad 129: 2120-2125$

362 Rice RH, Bradshaw KM, Durbin-Johnson BP, Rocke DM, Eigenheer RA, Phinney BS,

363 Sundberg JP (2012) Differentiating inbred mouse strains from each other and those with single

364 gene mutations using hair proteomics. PLoS One 7:e51956

365 Rice RH, Bradshaw KM, Durbin-Johnson BP, Rocke DM, Eigenheer RA, Phinney BS,

366 Schmuth M, Gruber R (2013) Distinguishing ichthyoses by protein profiling. PLoS One

367 8(10):e75355

368 Robinson MD, Oshlack A (2010) A scaling normalization method for differential expression

369 analysis of RNA-seq data. Genome Biology 11:R25

370 Rowe WF (2001) The current status of microscopical hair comparisons. The Scientific

371 World Journal 1:868-878

372 Schweizer J, Langbein L, Rogers MA, Winter H (2007) Hair follicle-specific keratins and

373 their diseases. Experimental Cell Research 313:2010-2020 
375 of Forensic Science 54:1198-1202

376 Shimomura Y, Wajid M, Zlotogorskic A, Lee YJ, Rice RH, Christiano AM (2009) Founder

377 mutations in the lipase H (LIPH) gene in families with autosomal recessive woolly

378 hair/hypotrichosis. Journal of Investigative Dermatology 129:1927-1934

379 Stark HJ, Breitkreutz D, Limat A, Ryle CM, Roop D, Leigh I, Fusenig N (1990) Keratins 1

380 and 10 or homologues as regular constituents of inner root sheath and cuticle cells in the human

381 hair follicle. European Journal of Cell Biology 52:359-372

382 Swift JA, Bews B (1974a) The chemistry of human hair cuticle II: The isolation and amino

383 acid analysis of the cell membranes and A-layer. Journal of the Society of Cosmetic Chemists

$384 \quad 25: 355-366$

385 Swift JA, Bews B (1974b) The chemistry of human hair cuticle - I: A new method for the

386 physical isolation of cuticle. Journal of the Society of Cosmetic Chemists 25:13-22

387 Taupin JM (2004) Forensic hair morphology comparison - a dying art or junk science?

388 Science \& Justice 44:95-100

389 Thibaut S, Candi E, Pietroni V, Melino G, Schmidt R, Bernard BA (2005) Transglutaminase

3905 expression in human hair follicle. J Invest Dermatol 125:581-585

391 Zahn H, Fohles J, Nienhaus M, Schwan A, Spel M (1980) Wool as a biological composite

392 structure. Industrial Engineering and Chemical Product Research Development 19:496-501

393 Zhang Y, Wen Z, Washburn MP, Florens L (2010) Refinements to label free proteome

394 quantitation: how to deal with peptides shared by multiple proteins. Analyt Chem 82: 2272-2281 


\section{Table $\mathbf{1}_{\text {(on next page) }}$}

Pairwise comparisons of 76 proteins in profiles from samples in each of four ethnic groups.

Values are number of proteins that were significantly different in weighted spectral counts.

Subjects in each group - six Caucasian and five each African-American (African-Amer), Kenyan and Korean - are numbered and labeled CA, AA, KE and KO, respectively. 


$\begin{array}{llllll}\text { Caucasian } & \text { CA2 } & \text { CA3 } & \text { CA4 } & \text { CA5 } & \text { CA6 } \\ \text { CA1 } & 7 & 5 & 11 & 15 & 26 \\ \text { CA2 } & & 4 & 16 & 13 & 27 \\ \text { CA3 } & & & 14 & 23 & 23 \\ \text { CA4 } & & & & 23 & 34 \\ \text { CA5 } & & & & & 30\end{array}$

African-Amer AA2 AA3 AA4 AA5

$\begin{array}{lllll}\text { AA1 } & 26 & 22 & 24 & 18\end{array}$

$\begin{array}{llll}\text { AA2 } & 18 \quad 20 \quad 20\end{array}$

AA3 56

AA4 9

$\begin{array}{lllll}\text { Kenyan } & \text { KE2 } & \text { KE3 } & \text { KE4 } & \text { KE5 } \\ \text { KE1 } & 6 & 14 & 13 & 16 \\ \text { KE2 } & & 10 & 5 & 21 \\ \text { KE3 } & & & 22 & 22 \\ \text { KE4 } & & & & 18\end{array}$

$\begin{array}{lllll}\text { Korean } & \text { KO2 } & \text { KO3 } & \text { KO4 } & \text { KO5 } \\ \text { KO1 } & 2 & 3 & 37 & 39 \\ \text { KO2 } & & 4 & 40 & 40 \\ \text { KO3 } & & & 28 & 32 \\ \text { KO4 } & & & & 21\end{array}$




\section{Figure 1}

Weighted spectral counts for nine proteins differentially expressed in hair shafts from six Caucasian subjects.

Samples were analyzed separately from three locations on the scalp. 
K32

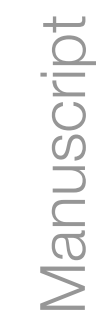

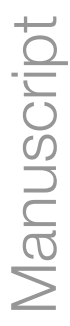

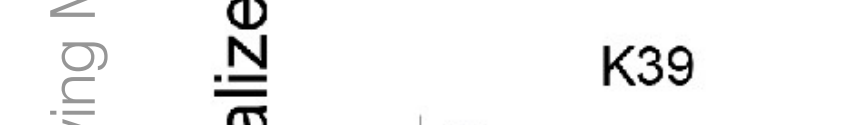

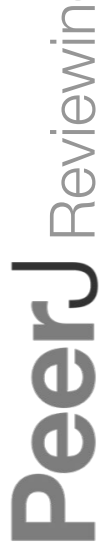
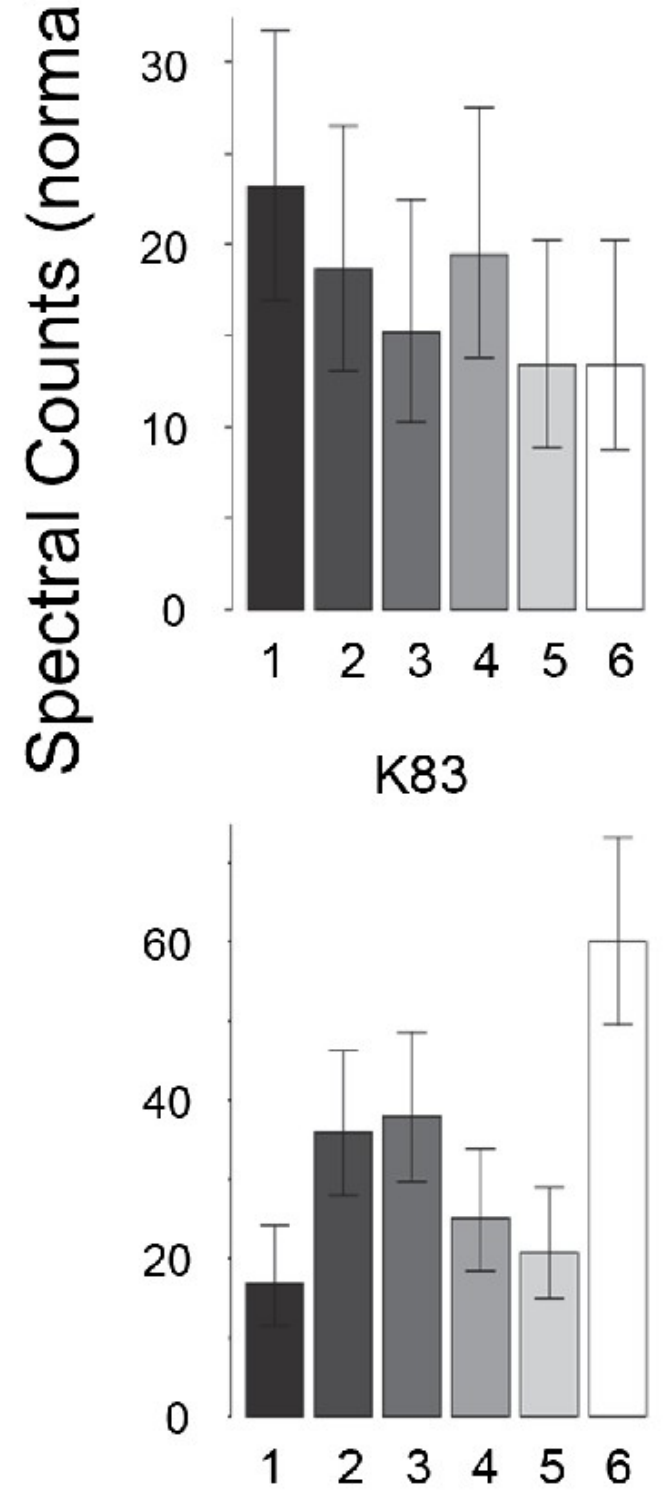

$\mathrm{K} 33 \mathrm{~A}$

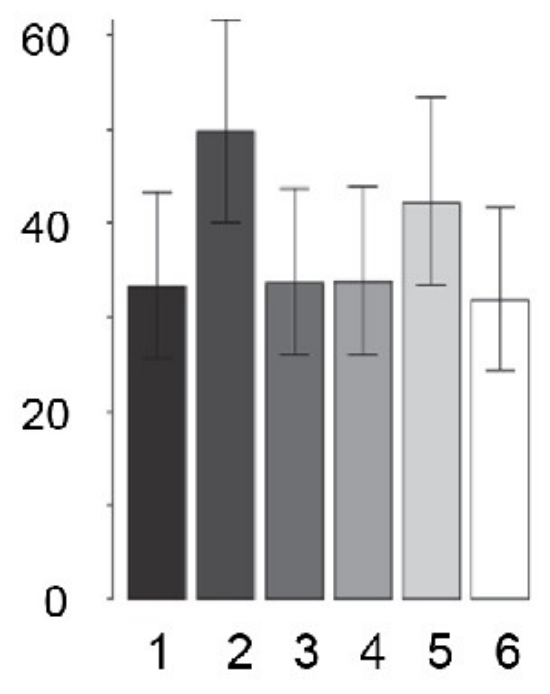

$\mathrm{K} 81$
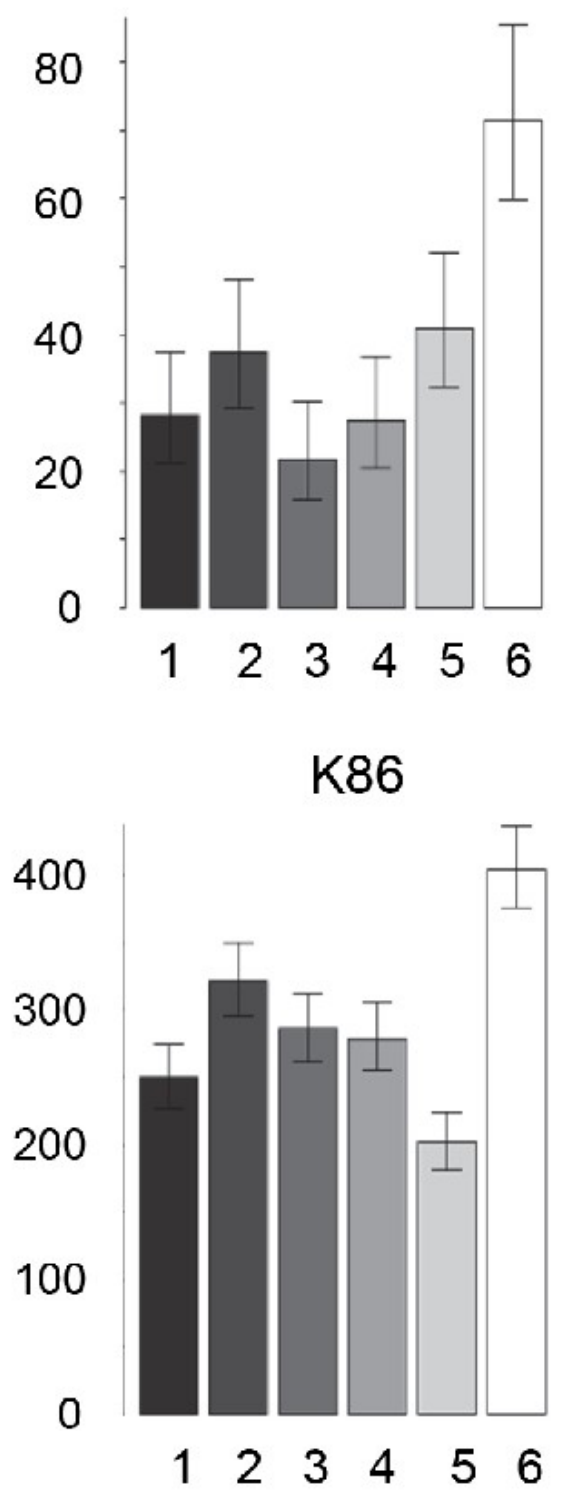

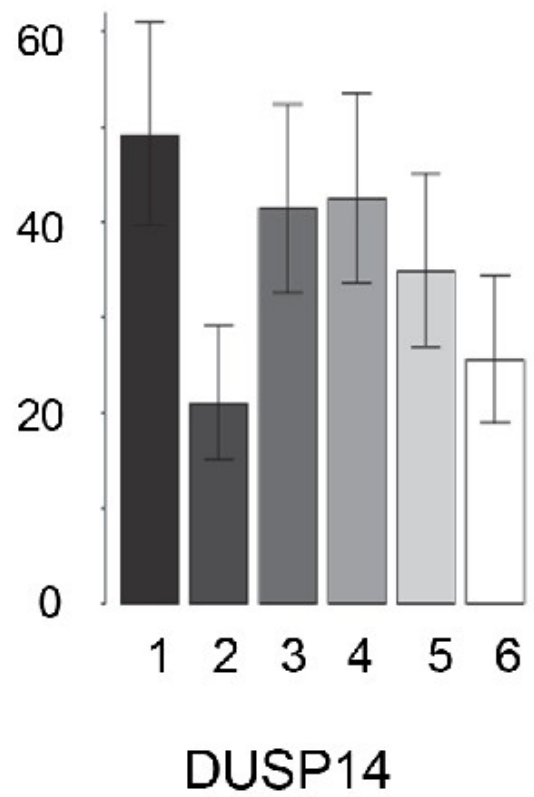

K35

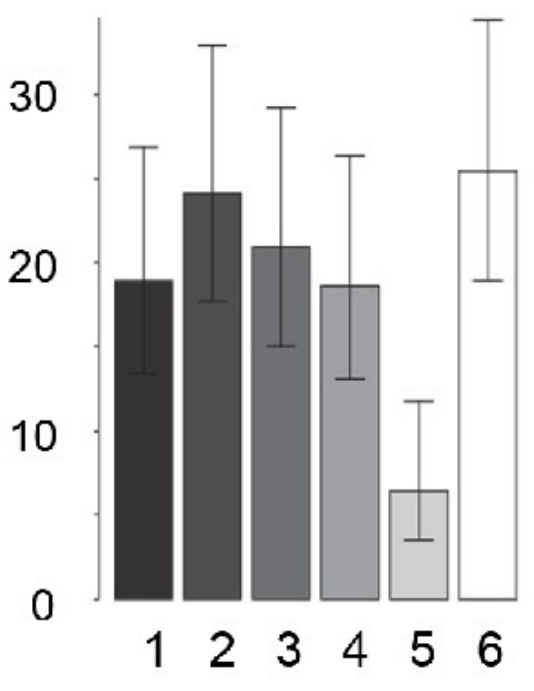

$\mathrm{K} 82$

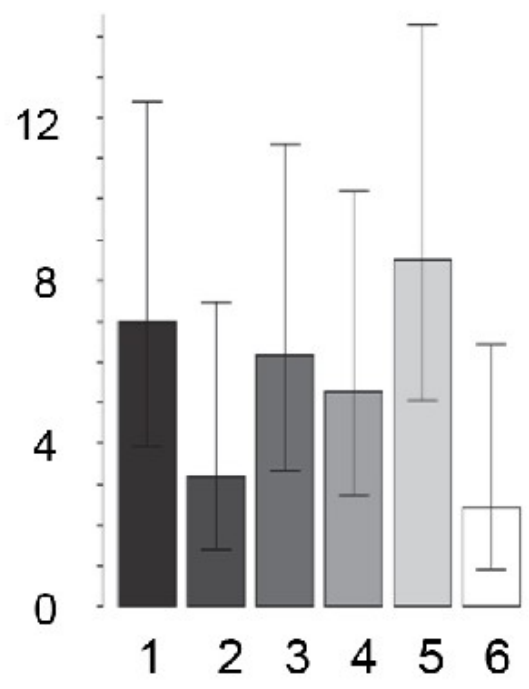




\section{Table 2 (on next page)}

Pairwise comparisons of proteins in hair from subjects from different ethnic groups.

Values are significant differences in weighted spectral counts from hair from African-American $(\mathrm{AA})$, Caucasian (CA), Kenyan (KE) and Korean (KO) subjects. 


$\begin{array}{llll} & \text { CA } & \text { KE } & \text { KO } \\ \text { AA } & 8 & 6 & 7 \\ \text { CA } & & 4 & 3 \\ \text { KE } & & & 4\end{array}$

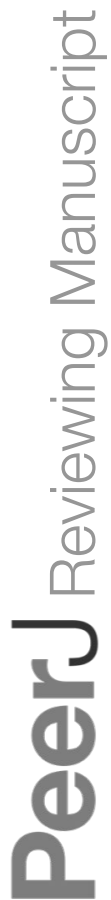

PeerJ reviewing PDF | (v2014:06:2249:1:0:NEW 17 Jul 2014) 


\section{Figure 2}

Weighted spectral counts for six proteins differentially expressed in hair from subjects of different ethnic origin.

Each bar represents the aggregate for Caucasian (CA), African-American (AA), Kenyan (KE) and Korean (KO) samples.

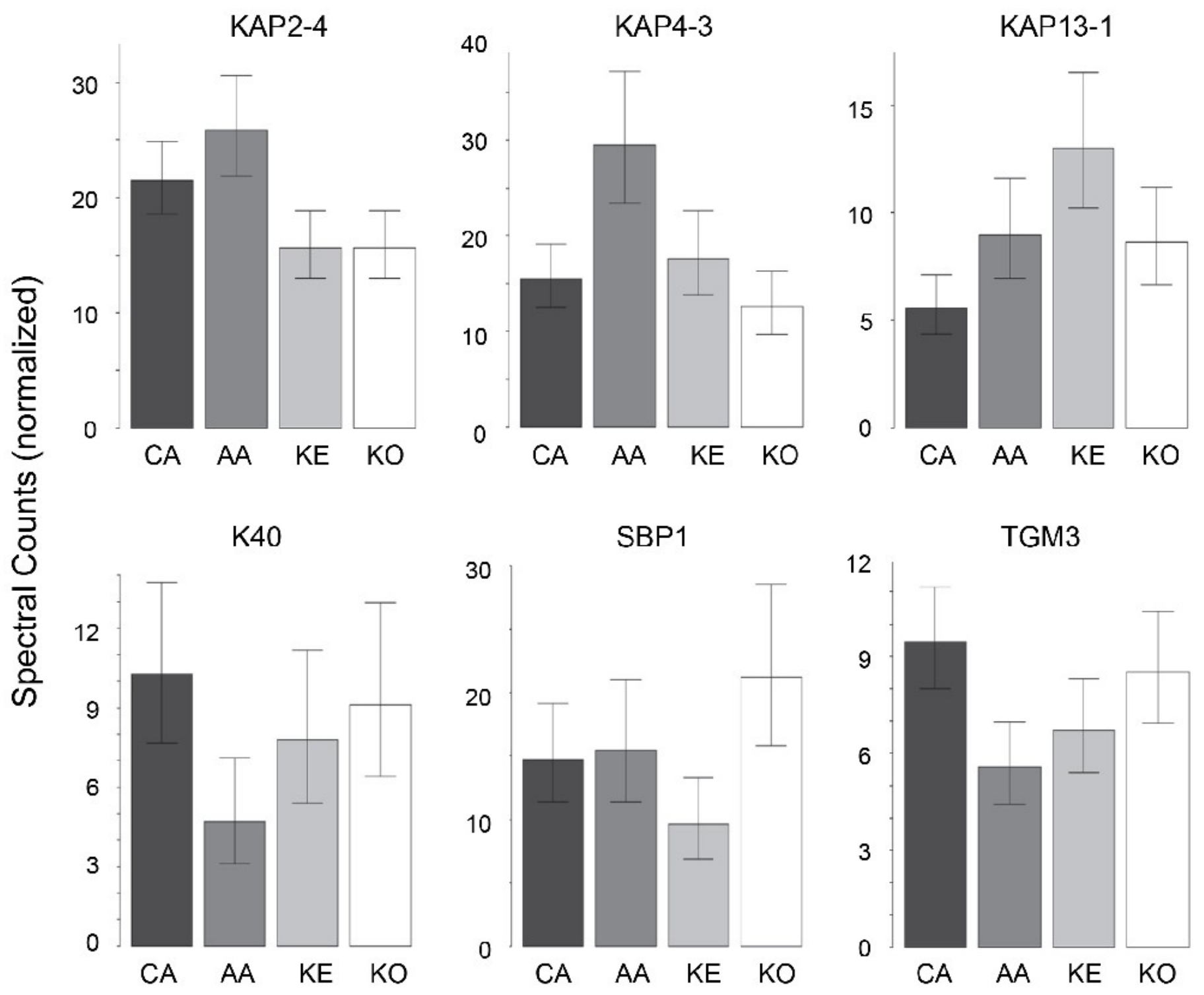




\section{Figure 3}

Pairwise comparisons of proteins (92) in hair obtained from different body sites of the same four individuals.

The values shown for significantly different weighted spectral counts were used for hierarchical clustering by relatedness. 


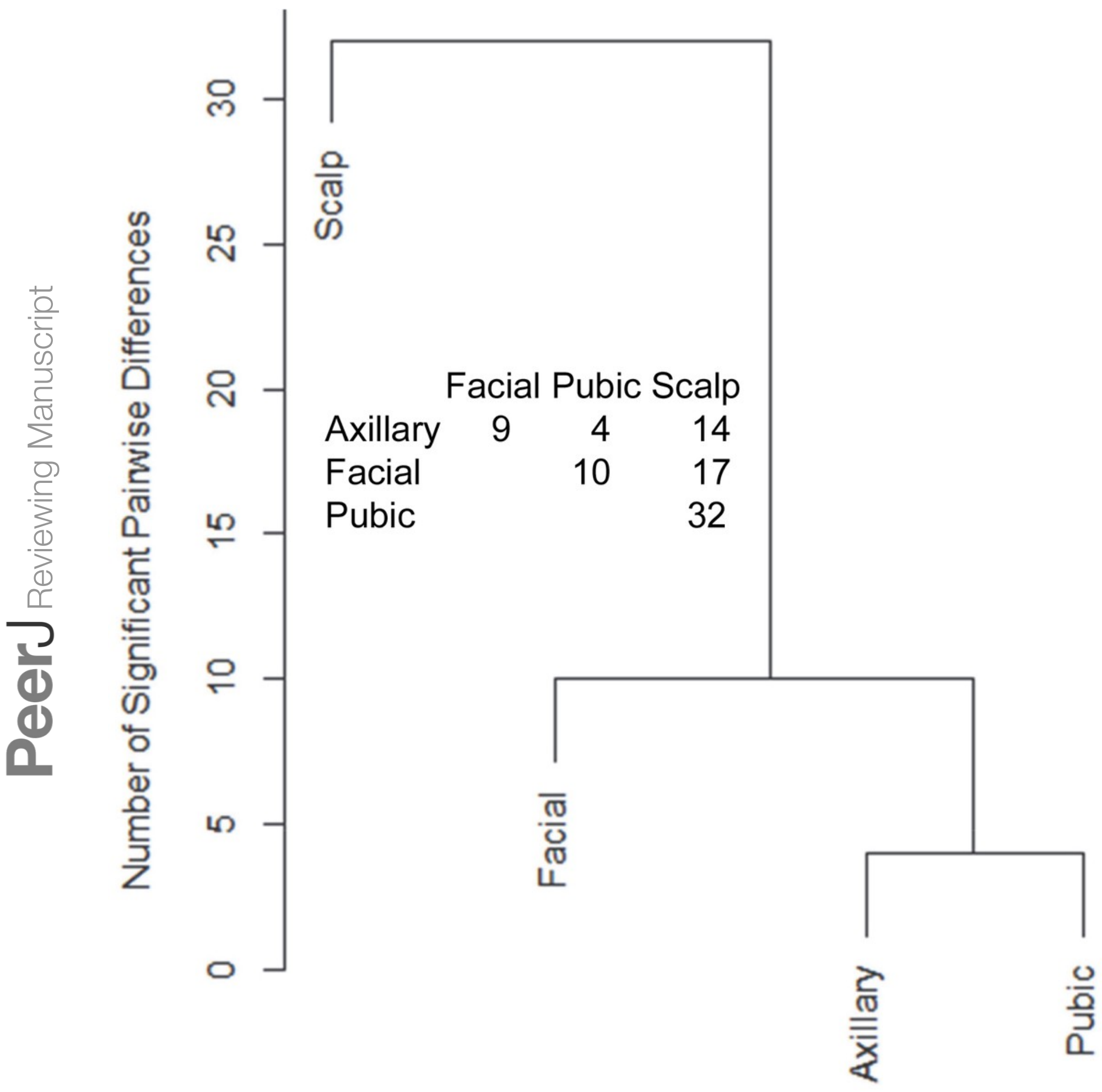




\section{Figure 4}

Weighted spectral counts for 10 proteins differentially expressed in hair from different body sites of Caucasian subjects.

Illustrated are composite values for single samples of Ax (axillary), $\mathrm{Fa}$ (facial), and $\mathrm{Pu}$ (pubic) hair from each of four Caucasian subjects and for triplicate values of Sc (scalp) hair from the same four subjects.

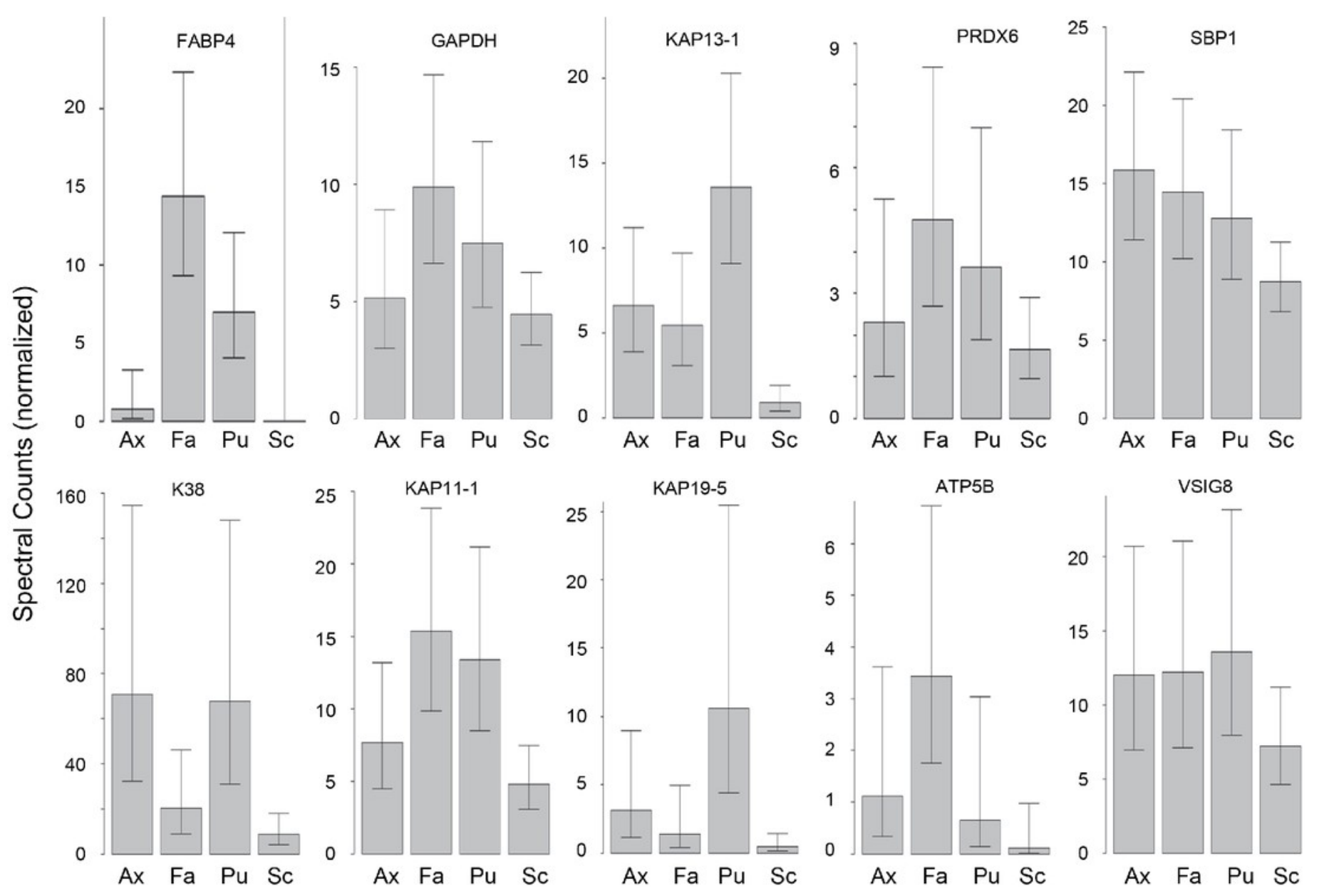




\section{Figure 5}

Example of cuticle fractions isolated for proteomic analysis.

The upper panel shows a sample of purity $>95 \%$ cuticle cells used in the analysis, while the lower panel (not used) had an estimated purity of $70 \%$. The vertical white hash marks at the bottom of each panel mark $10 \mu \mathrm{m}$ intervals. 


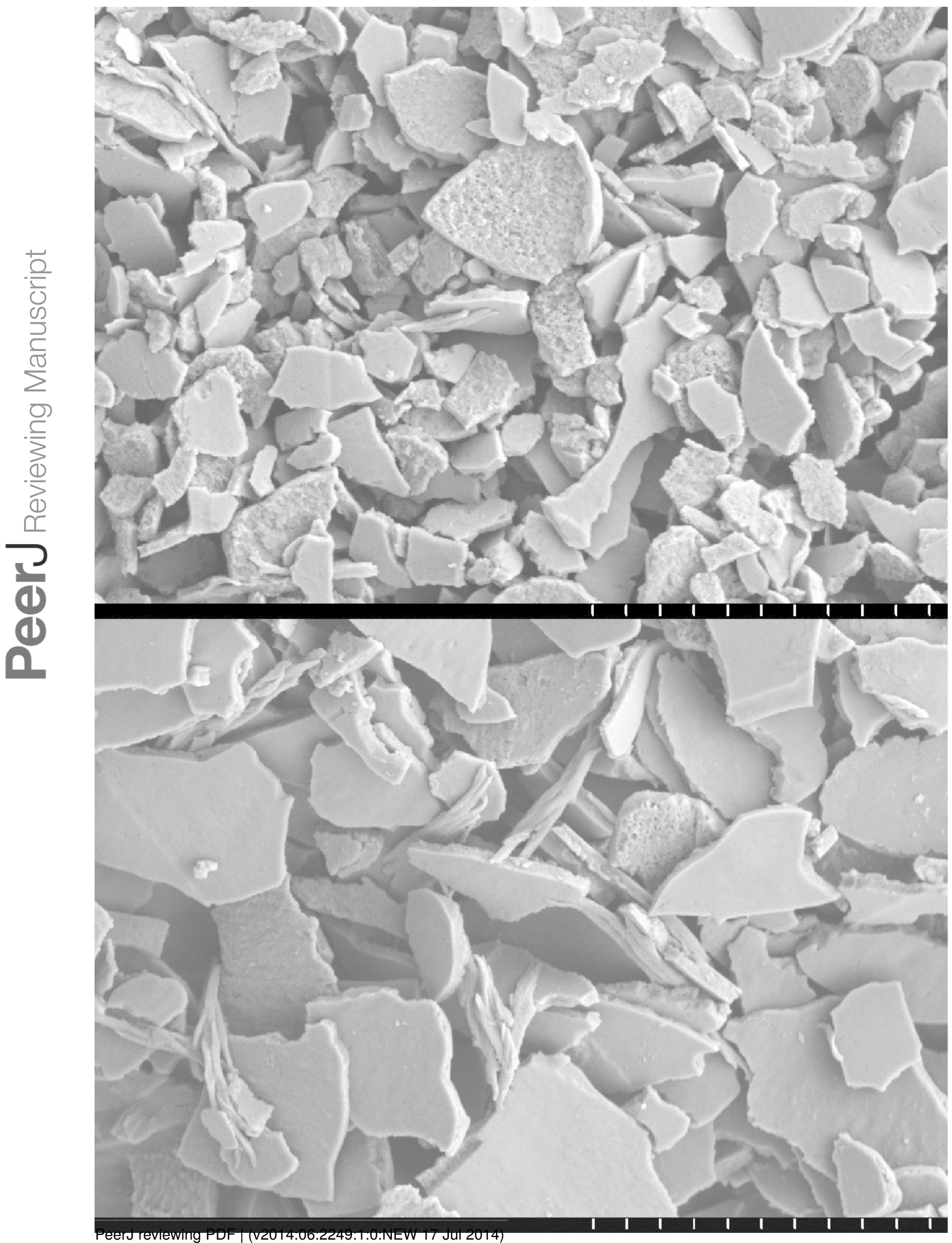




\section{Figure 6}

\section{Differentially expressed proteins.}

Illustrated are eight proteins whose weighted spectral counts are significantly higher in cuticle (Cut), shown in the upper row, and eight with higher levels in the total hair shaft (Tot) shown in the lower row. These compare levels in the same five subjects.

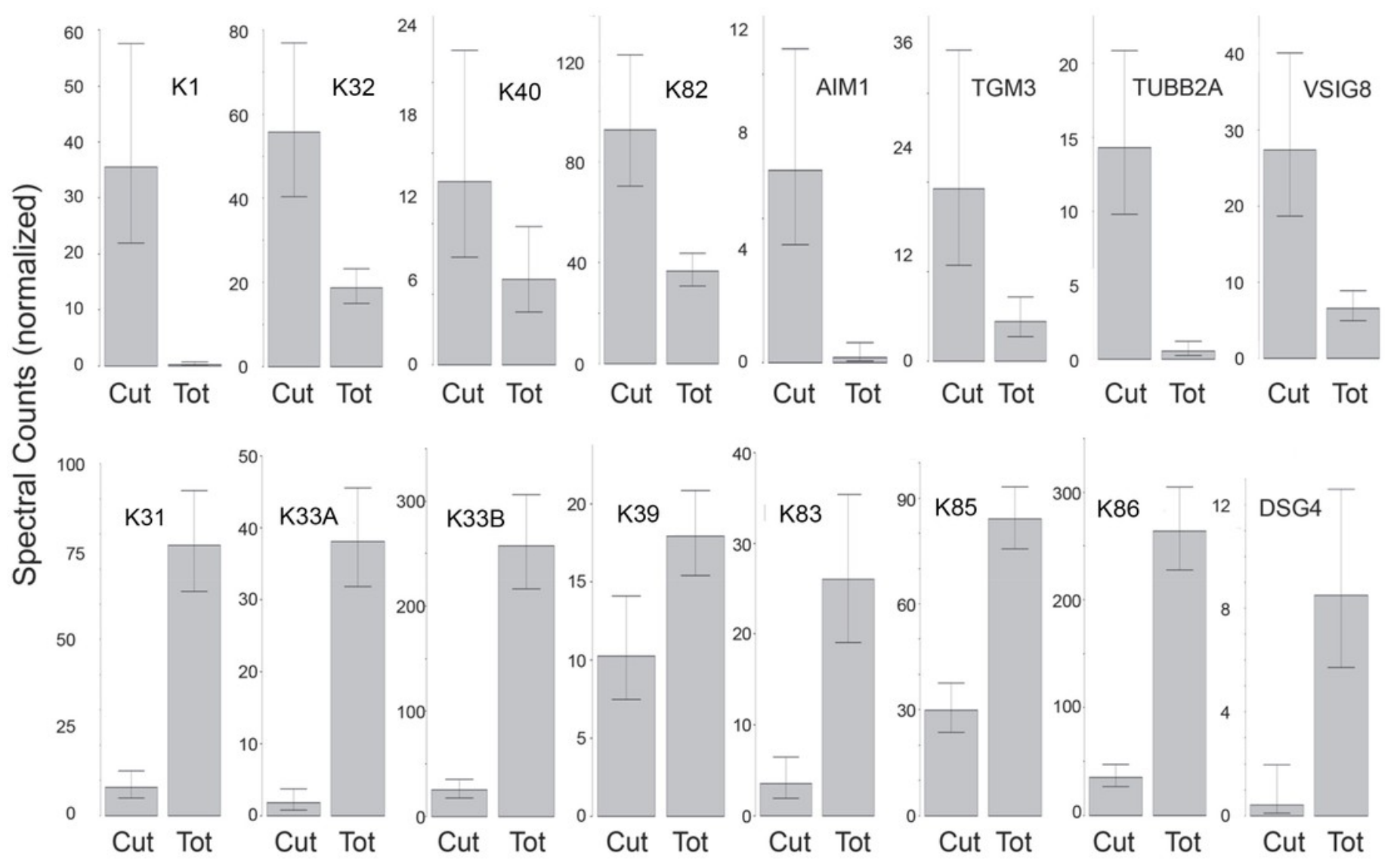

